\title{
Serum Irisin, Adropin, and Preptin in Obese Patients 6 Months After Bariatric Surgery
}

\author{
M. Glück ${ }^{1}$ (D) • J. Glück ${ }^{2}$ - M. Wiewióra ${ }^{1}$ • B. Rogala ${ }^{2}$ • J. Piecuch ${ }^{1}$
}

Published online: 17 June 2019

(C) The Author(s) 2019

\begin{abstract}
Background/Objectives The reduction of body mass after bariatric surgery affects energy metabolism and may involve changes in irisin, preptin, and adropin production.

Subjects and Methods Fifty-five morbidly obese patients with a mean body mass index (BMI) of $45.7 \pm 5.8 \mathrm{~kg} / \mathrm{m}^{2}$ were treated with either laparoscopic sleeve gastrectomy $(n=30)$ or laparoscopic adjustable gastric banding $(n=25)$. Forty-six $(83.6 \%)$ were followed-up 6 months after surgery. The control group included 15 healthy non-obese participants. Anthropometric measurements, lipid profiles, $\mathrm{HbA1c}$, and serum irisin, preptin, and adropin were assessed at baseline and on follow-up.

Results The serum concentrations of all three peptides were higher at 6 months than at baseline but only irisin $(p=0.02)$ and adropin $(p=0.000001)$ were significantly higher. The increase in preptin was borderline significant $(p=0.051)$. Changes of serum concentrations of all three peptides were bidirectional.

Conclusion Body mass reduction resulting from bariatric procedures may change the production of energy regulating peptides, but not always in a favorable manner.
\end{abstract}

Keywords Obesity $\cdot$ Bariatric surgery $\cdot$ Irisin $\cdot$ Adropin $\cdot$ Preptin

\section{Introduction}

Metabolic changes and pathophysiological effects that occur following the reduction in body mass reduction produced by

M. Glück

marekgluck46@gmail.com

J. Glück

joagluck@mp.pl

M. Wiewióra

m-wiewiora@tlen.pl

B. Rogala

b_rogala@pro.onet.pl

J. Piecuch

jerzy.piecuch@wp.pl

1 Department of General and Bariatric Surgery and Emergency Medicine, Medical University of Silesia, Zabrze, Poland

2 Department of Internal Medicine, Allergology and Clinical Immunology, Medical University of Silesia, Katowice, Poland bariatric surgery are not fully understood. Cytokines are produced and secreted by various organs, and those produced by skeletal muscle have been known for some time $[1,2]$. Myokines are secreted upon physical activity and may have positive effects on metabolic disorders by increasing glucose uptake and distribution, enhancing lipolysis, and influencing angiogenesis and revascularization. Irisin is a myokine derived from fibronectin type III domain-containing protein 5 (FNDC5), which is highly expressed in skeletal muscle [3], and synthesis is induced by physical activity [4]. Irisin is a messenger that promotes the conversion of lipid-storing white adipose to energy-catabolizing brown adipose tissue that leads to increased thermogenesis. Irisin may thus protect against obesity and diabetes. In an animal model of diabetes, irisin improved glucose tolerance, glucose uptake by myocytes, and reduced the expression of two liver enzymes that stimulate gluconeogenesis. It also reduced the fat percentage of body weight, as well as serum total cholesterol and triglycerides [5].

Reports of the influence of exercise training on FNDC5 gene expression in human muscle by exercise training are inconsistent. Boström et al. found that exercise training induced the expression of the FNDC5 gene in human muscle, which was associated with increased irisin expression [4]. 
However, Timmons et al. reported that FNDC5 expression increased only in highly active elderly subjects and was not associated with metabolic status [6]. In the general population, irisin concentration increases with routine daily physical activity and circulating irisin is associated with a favorable lipid profile $[7,8]$. Studies investigating serum irisin in obese individuals are equivocal. A study including 300 obese participants found that their serum irisin levels were not significantly higher than those in age- and sex-matched controls [9]. Other studies reported lower serum irisin levels in obese than in normal-weight participants, but that the difference was associated with the presence or absence of diabetes mellitus [10-12]. There are limited studies on a change of serum irisin levels as a result of body mass reduction. Fukushima et al. did not find a significant association of serum irisin level and weight loss in participants in a 6-month weight program including diet intervention and exercise and cognitive behavioral therapy, and concluded that body weight reduction did not change irisin levels [13]. Studies on the impact of bariatric surgery on serum irisin had protocols involved Roux-en-Y gastric bypass or sleeve gastrectomy and had different lengths of post-surgical follow-up. The results are inconsistent, reporting either stable levels at 3,6, and 12 months [11, 14], or bidirectional changes with increases or decreases at 1 month after surgery [15].

Two other cytokines that regulate energy metabolism are preptin and adropin, but less is known of their roles in obesity than is known about irisin. Preptin is a 34 amino-acid peptide hormone that is co-secreted with insulin and amylin by pancreatic beta-cells. It is believed to enhance glucose-mediated insulin secretion [16]. Elevated serum preptin has been reported in overweight and obese adults [17], but we have not found any published data on changes in preptin synthesis following body mass reduction.

Adropin is synthesized in the liver, brain, and many peripheral tissues [2], and is believed to control fatty-acid metabolism, prevent insulin resistance, and regulate dyslipidemia and impaired glucose tolerance [18]. Adropin expression is diet dependent. Serum adropin is reported to be lower in overweight and obese than in normalweight adults and to increase after Roux-en-Y gastric bypass, peaking 3 months after surgery [19].

Data on the influence of body mass reduction resulting from bariatric surgery on serum irisin, preptin, and adropin in humans are scarce and ambiguous [20]. The study design assumed that production of irisin, preptin, and adropin are impaired in obesity and that normal levels are restored after bariatric surgery. The levels of these three peptides were determined before surgery and 6 months after surgery taking changes in the body mass index (BMI) and serum lipid profile into account. The results in the surgical patients were compared with the corresponding values in a group of normalweight control participants.

\section{Material and Methods}

\section{Study Design, Settings, and Participants}

This single-center prospective observational case-control study included consecutive patients referred to the Department of General and Bariatric Surgery and Emergency Medicine between 2015 and 2016. The study was approved by the Local Ethics Committee at the Medical University of Silesia. All subjects gave informed written consent. Eligible patients were morbidly obese with a BMI > $35 \mathrm{~kg} / \mathrm{m}^{2},>18$ years of age, and indicated for bariatric surgery. All surgical procedures were performed by the same two surgeons at the same institution. Controls with BMIs between 18.5 and $25 \mathrm{~kg} / \mathrm{m}^{2}$ were recruited at the same department as the patients or at the Department of Internal Medicine, Allergology, and Clinical Immunology. Patients and control participants with chronic inflammatory conditions such as coronary heart disease, renal insufficiency, chronic pancreatitis, and acute or chronic inflammatory conditions such as acute hepatitis or pancreatitis, or coexisting malignancies were excluded.

\section{Patient Variables}

Obese patients were examined 1 or 2 days before surgery and 6 months after surgery during routine follow-up in the outpatient clinic. Baseline body weight, BMI, excess body weight (EBW), waist-hip ratio, total cholesterol (mg/dl), HDL cholesterol (mg/dl), LDL cholesterol (mg/dl), triglycerides $(\mathrm{mg} /$ $\mathrm{dl})$, glycated hemoglobin $(\mathrm{HbAlc})$, serum irisin, preptin, and adropin were assayed in cases and in controls. The same variables were assessed 6 months after surgery.

\section{Serum Irisin, Preptin, and Adropin Assays}

Commercial enzyme-linked immunosorbent assay kits were used to measure serum of irisin (BioVendor), adropin, and preptin (both Cloude-Clone Corp). The assays were performed following the manufacturers' instructions. The lower limits of detection were $1 \mathrm{ng} / \mathrm{ml}$ for irisin, $13.7 \mathrm{pg} / \mathrm{ml}$ for adropin, and $15.3 \mathrm{pg} / \mathrm{ml}$ for preptin. Values lower than the sensitivity limit were assigned values by extrapolation of the standard curves generated for each set of sample assays.

\section{Statistical Analysis}

Patient baseline characteristics and operative outcomes were evaluated by descriptive statistics. Quantitative variables were reported as means \pm standard deviation if normally distributed, or medians and interquartile range if not normally distributed. Qualitative data were reported as frequencies and percentages. Ranges were also reported. Peptide concentrations were 
reported as medians and interquartile ranges because of the non-normal distribution of preptin and adropin values before surgery and of irisin and preptin at the 6-month follow-up. Qualitative variables were compared by the chi-squared test. Quantitative variables were compared by Student's $t$ test if they were normally distributed, or the Mann-Whitney $U$ test and Wilcoxon signed-rank test if not normally distributed. Multiple regression analysis was performed with age, sex, BMI, EBW, serum glucose, total cholesterol, HDL cholesterol, LDL cholesterol, triglycerides, and $\mathrm{HbAlc}$ to determine the association with serum peptide concentration before and after surgery. The statistical analysis was performed with Statistica 12.5 (https://www.tibco.com/products/datascience). $p$ values $<0.05$ were considered significant.

\section{Results}

\section{Patient Characteristics}

Fifty-five morbidly obese patients, 45 women $(82 \%)$ and ten men $(12 \%, p<0.0001)$, age $42.4 \pm 9.7$ years and BMI $45.7 \pm$ 5.7 (range $36.3-62.5) \mathrm{kg} / \mathrm{m}^{2}$, with bariatric surgery were evaluated. Twenty-five (45\%) experienced laparoscopic sleeve gastrectomy (LSG) and 30 (54\%) experienced laparoscopic adjustable gastric banding (LAGB, $p>0.05$ ). LSG patients were $44.9 \pm 9.1$ years of age and had a BMI of $49.9 \pm$ $5.3 \mathrm{~kg} / \mathrm{m}^{2}$. Eighteen (72\%) were women. LABG patients were $40.3 \pm 9.87$ years of age and had a BMI of $42.2 \pm 3.3 \mathrm{~kg} / \mathrm{m}^{2}$ $(p<0.0001)$. Twenty-seven $(90 \%)$ were women. Forty-six $(83.6 \%)$ patients were re-evaluated 6 months after surgery. The control group included 15 non-obese, age-matched participants, seven women (46.7\%) with a mean age of $38.7 \pm$ 6 years and BMI of $23.2 \pm 2.1 \mathrm{~kg} / \mathrm{m}^{2}$. The patient characteristics at baseline and at 6 months after surgery are shown in Table 1. Six months after surgery, body weight, BMI, and EBW were significantly reduced compared with baseline. EBW was $41.2 \pm 11.9 \%$. HbAlc and triglycerides were significantly lower than baseline, total cholesterol levels did not differ, and both LDL- and HDL cholesterol significantly increased (Table 1).

Serum irisin, preptin, and adropin were assayed at baseline and at 6 months after surgery. Baseline serum irisin was significantly lower in obese patients than in healthy controls and adropin and preptin levels in obese patients were comparable with those in controls. Of all three peptides increased in the 6 months after surgery, the difference was significant only for irisin $(p=0.02)$ and adropin $(p=0.000001)$. The difference in preptin level was borderline ( $p=0.051$, Table 2 and Figs. 1 and 2). The changes were bidirectional. Irisin and preptin both decreased in $19(41 \%)$ patients and both increased in 27 $(59 \%)$. Adropin decreased in six patients (13\%) and increased in $40(87 \%$, Table 3$)$.
Comparison of patients with increased versus decreased irisin, preptin, or adropin concentrations at 6 months revealed that the baseline serum peptide values in the two subgroups were different (Table 3). Patients with a rise in serum irisin at 6 months had significantly lower baseline serum irisin $(p=$ 0.001 ) and lower HbAlc than those with a decrease at 6 months ( $p=0.023$, Fig. 2a). Patients with rise in serum preptin at 6 months had significantly lower baseline serum preptin ( $p=0.002$, Fig. 2b). Patients with a rise in serum adropin at 6 months had significantly lower baseline serum adropin $(p=0.0018)$ and were younger $(p=0.018$, Fig. $2 \mathrm{c})$. Serum irisin, preptin, and adropin values at baseline and follow-up in LABG patients were not significantly different from those in LSG patients (data not shown).

Multiple regression including age, sex, BMI, EBW, serum glucose, total cholesterol, HDL cholesterol, LDL cholesterol, triglycerides, and $\mathrm{HbA1c}$ found that $\mathrm{EBW}, \mathrm{HbA1c}$, total cholesterol, LDL cholesterol, and triglycerides were associated with preoperative serum irisin $(p=0.0064)$, but not adropin or preptin. Multiple regression analysis of postoperative peptides found that EBW and BMI were independently associated with serum preptin ( $p=0.02$ and 0.01 , respectively), but the association was not significant when all above-mentioned factors were taken together.

\section{Discussion}

In this study, serum irisin, preptin, and adropin in obese patients before and after bariatric surgery were compared with values in healthy normal-weight control participants. Serum irisin at baseline was significantly lower in obese patients than in control participants, but the difference was no longer significant 6 months after bariatric surgery. At 6 months, both serum irisin and adropin had significantly increased. Preptin also increased, but the difference from baseline was of borderline significance. The changes from before to after surgery were bidirectional. For each of the three peptides, the baseline value before surgery was significantly associated with the subsequent increase or decrease in serum level after surgery. The study results are in line with those reported by Lee et al. who reported bidirectional changes 1 month after Roux-en-Y gastric bypass surgery, with elevations of serum irisin at 9 months, but not at 1 month, associated with lower preoperative levels. Preptin and adropin were not studied [16]. Our results and those of Lee et al. indicate that preoperative serum levels of peptides like irisin influence the increase that occurs in response to the postoperative reduction in body mass. In this study, the preoperative $\mathrm{HbAlc}$ percentages in patients with increased and decreased postoperative serum irisin were different. Preoperative $\mathrm{HbAlc}$ was significantly higher in patients with high preoperative serum irisin that decreased following surgery, indicating that impaired glucose metabolism 
Table 1 Characteristics of the obese study population at baseline and at 6 months after surgery

\begin{tabular}{|c|c|c|c|}
\hline Characteristics & Median and IQR or mean $\pm \mathrm{SD}$ & Range & $p$ value \\
\hline Age (years) & $42.4 \pm 9.7$ & $21-61$ & \\
\hline \multicolumn{4}{|l|}{ Weight (kg) } \\
\hline Baseline & $128.8 \pm 17.8$ & $95-163$ & \multirow[t]{2}{*}{$p<0.00001$} \\
\hline 6 months postoperative & $101 \pm 13.5$ & $69-127$ & \\
\hline \multicolumn{4}{|l|}{ BMI $\left(\mathrm{kg} / \mathrm{m}^{2}\right)$} \\
\hline Baseline & $45.7 \pm 5.7$ & $36.3-62.5$ & \multirow[t]{2}{*}{$p<0.00001$} \\
\hline At 6 months & $35.8 \pm 3.9$ & $27.3-43.6$ & \\
\hline \multicolumn{4}{|l|}{ EBW } \\
\hline Baseline & $67.8 \pm 15.8$ & $42.8-101.8$ & \multirow[t]{2}{*}{$p<0.00001$} \\
\hline At 6 months & $41.4 \pm 14.3$ & $(15.9-104)$ & \\
\hline \multicolumn{4}{|l|}{ WHR } \\
\hline Baseline & $0.8 \pm 0.05$ & $0.79-1.01$ & \multirow[t]{2}{*}{$p=0.000009$} \\
\hline At 6 months & $0.92 \pm 0.06$ & $0.8-1.1$ & \\
\hline \multicolumn{4}{|l|}{$\%$ EWL } \\
\hline At 6 months & $41.2 \pm 11.9$ & $7.1-68.7$ & \\
\hline \multicolumn{4}{|l|}{ HbA1c (\%) } \\
\hline Baseline & $5.34(5.2-6.2)$ & $4.9-9.5$ & \multirow[t]{2}{*}{$p<0.0004$} \\
\hline At 6 months & $5.4(5.1-5.6)$ & $4.7-7.8$ & \\
\hline \multicolumn{4}{|l|}{ Glucose fasting (mg/dl) } \\
\hline Baseline & $5.34(4.94-6.04)$ & $4.31-9.64$ & \multirow[t]{2}{*}{$p=0.28$} \\
\hline At 6 months & $5.67(5.18-6.12)$ & $4.48-10.9$ & \\
\hline \multicolumn{4}{|l|}{ Total cholesterol (mg/dl) } \\
\hline Baseline & $194(183.5-229.5)$ & $129-268$ & \multirow[t]{2}{*}{$p=\mathrm{ns}$} \\
\hline At 6 months & $208.5(178-223)$ & $106-303$ & \\
\hline \multicolumn{4}{|l|}{ HDL cholesterol } \\
\hline Baseline & $49(42-56)$ & $35-81$ & \multirow[t]{2}{*}{$p=0.003$} \\
\hline At 6 months & $53(48-59)$ & $36-123$ & \\
\hline \multicolumn{4}{|l|}{ LDL cholesterol } \\
\hline Baseline & $111.6 \pm 37.8$ & $38-177$ & \multirow[t]{2}{*}{$p=0.004$} \\
\hline At 6 months & $129.8 \pm 38.9$ & $11-207$ & \\
\hline \multicolumn{4}{|l|}{ Triglycerides (mg/dl) } \\
\hline Baseline & $146(105-187)$ & $58-575$ & \multirow[t]{2}{*}{$p=0.001$} \\
\hline At 6 months & $109.5(88-150)$ & $61-224$ & \\
\hline
\end{tabular}

$B M I$ body mass index, $E B W$ excess body weight, $W H R$ waist-hip ratio, $E W L$ excess weight loss, $H b A 1 c$ glycated hemoglobin, $H D L$ high-density lipoprotein, $L D L$ low-density lipoprotein may be related to irisin production and its change after reduction in body mass. A study by Shoukry et al. reported an association of irisin production and type 2 diabetes mellitus (T2DM), with a decrease in serum irisin in obese patients with T2DM but not in healthy controls and higher serum irisin in obese than in lean controls [12]. A systematic review and meta-analysis of 23 studies reported that circulating irisin concentrations were significantly lower in T2DM patients than in controls without T2DM [21]. If relatively low serum irisin has unfavorable effects on glucose and lipid metabolism, then the study results indicate that a history of T2DM may inhibit desirable effects of postoperative body mass reduction on irisin production. Preoperative serum irisin was lower in obese patients than in control participants, but at 6 months after surgery, the difference was no longer significant. Preoperative EBW, HbAlc, total cholesterol, LDL cholester$\mathrm{ol}$, and triglycerides levels were all significantly associated with serum irisin before surgery. The result is consistent with a role of irisin in lipid and glucose metabolism as discussed above; however, EBW was the only anthropometric variable that was associated with irisin level. The postoperative analysis found no significant associations of patient variables and irisin level. Several previous studies have investigated changes in serum irisin after body mass reduction. Fukushima et al. found no significant changes in serum irisin after body mass reduction resulting from diet, exercise therapy, and cognitive 
Table 2 Serum irisin, preptin, and adropin before and after bariatric in obese patients and control participants

\begin{tabular}{cclc}
\hline & Obese before operation & $\begin{array}{l}\text { Obese at } 6 \text { months after } \\
\text { operation }\end{array}$ & Controls \\
\hline Irisin $(\mu \mathrm{g} / \mathrm{ml})$ & $\begin{array}{c}1.54(1.24-1.75 ; \\
0.75-2.81)\end{array}$ & $1.7(1.42-2.03 ; 0.82-4.6)^{*}$ & $1.77(1.56-2.15 ; 1.19-3.3)^{\dagger}$ \\
Preptin & $\begin{array}{c}15.6(7.5-20.5 ; \\
(\mathrm{pg} / \mathrm{ml})\end{array}$ & $22(8.9-30.5 ; 3.2-54.6)$ & $9.2(5.2-14.06 ; 4.3-60.6)^{\ddagger}$ \\
Adropin & $255(212-327 ; 63-707)$ & $578(341-737 ; 131-1250)^{\S}$ & $\begin{array}{c}247.2(179.2-353.3 ; \\
(\mathrm{pg} / \mathrm{ml})\end{array}$ \\
\hline
\end{tabular}

Values are medians, interquartile range, and total range

CON controls

$* p=0.02$ vs. before surgery

${ }^{\dagger} p=0.02$ vs. obese before surgery

${ }^{\star} p=0.016$ vs. obese 6 months after surgery

${ }^{\S} p=0.00001$ vs. obese before surgery

${ }^{\eta} p=0.0001$ vs. obese at 6 months after surgery behavioral therapy [13]. However, the reduction in BMI of $2.4 \mathrm{~kg} / \mathrm{m}^{2}$ was less than $9.96 \pm 4.3 \mathrm{~kg} / \mathrm{m}^{2}$ in our study.
Demirpence et al. found no change in serum irisin after sleeve gastrectomy, but the follow-up was at 3 months, which was a

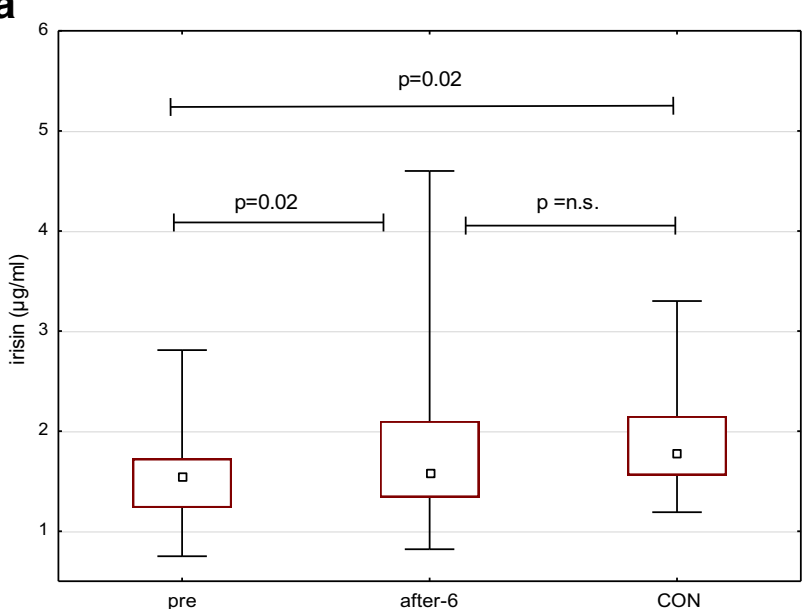

b

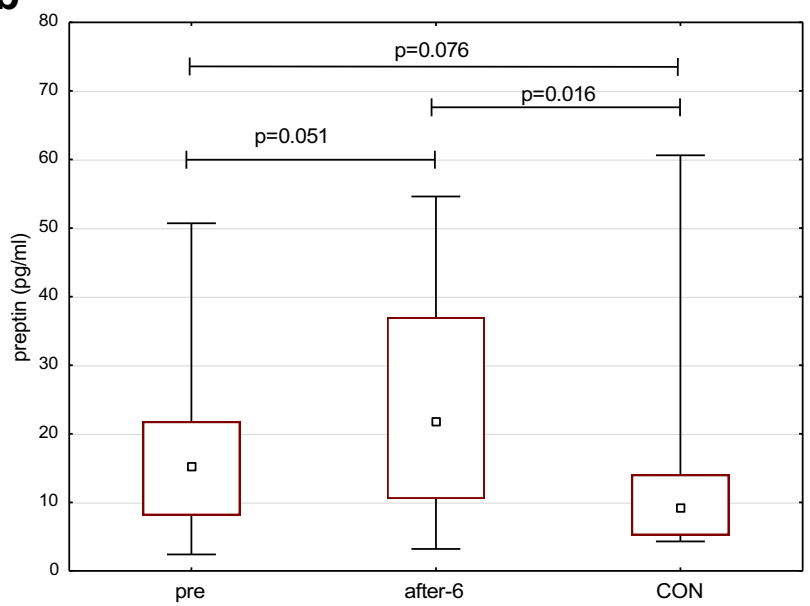

C

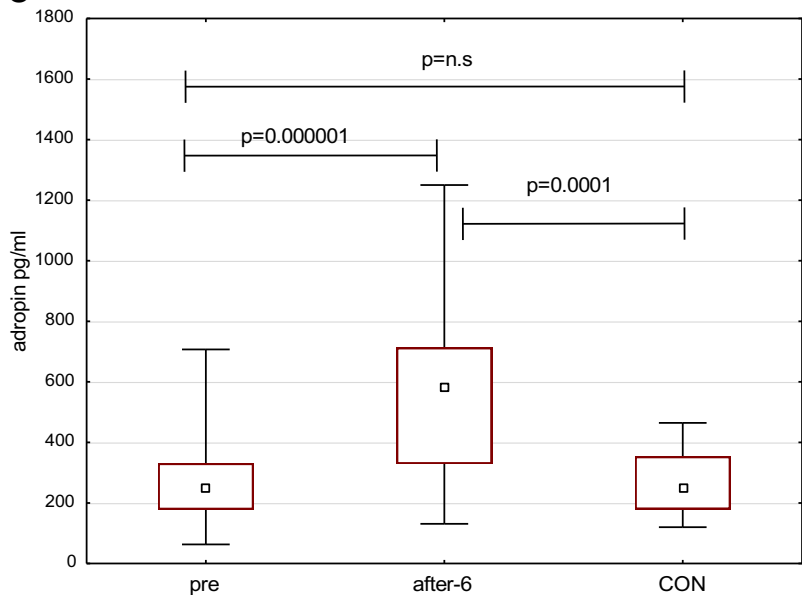

Fig. 1 Serum irisin (a), preptin (b), and adropin (c) in obese patients before surgery (pre), 6 months after surgery (after-6), and in controls (CON). Data are medians, interquartile, and total ranges 
a

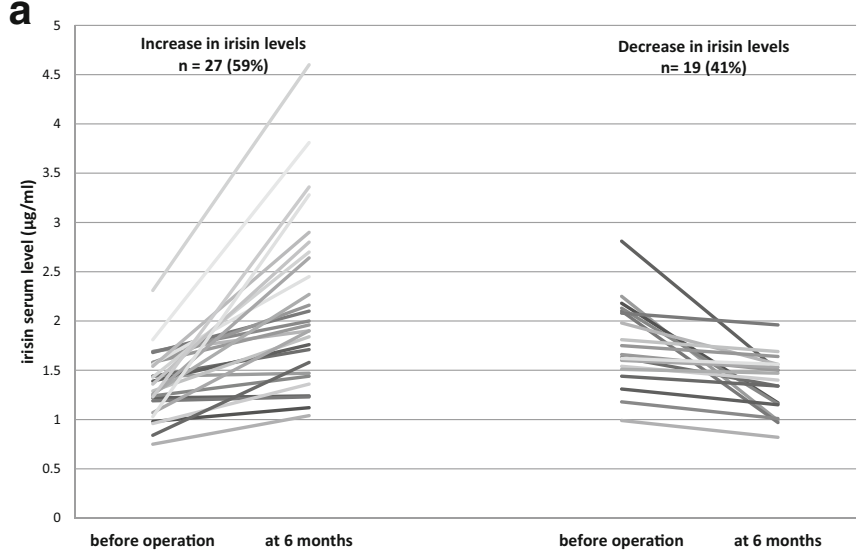

b

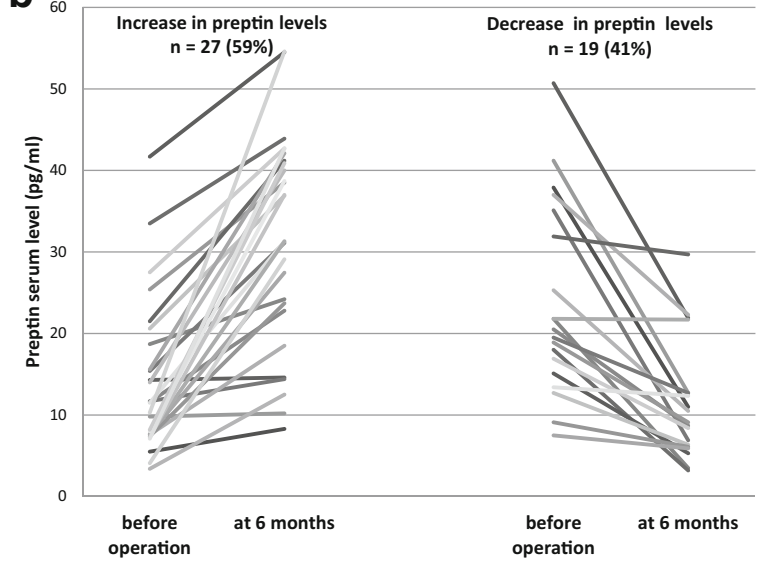

C

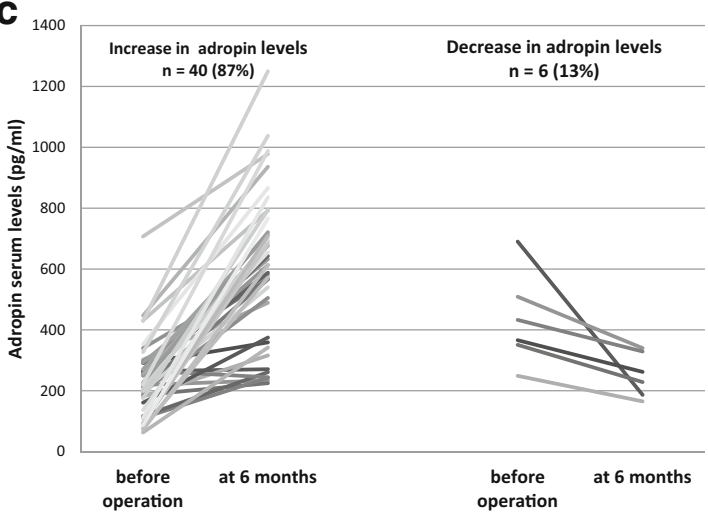

Fig. 2 Changes in irisin (a), preptin (b), and adropin (c) in obese patients stratified by the direction of serum-level change

shorter than the 6 months in our study [11]. Majorczyk et al. reported stable irisin levels at 6 and 12 months after LSG or laparoscopic Roux-en-Y gastric bypass [15]. Lee at al. found that preoperative serum irisin concentration was significantly correlated with the percentage of body weight lost in 1 year, suggesting that preoperative irisin might be at least in part associated with weight loss following bariatric surgery in morbidly obese patients [16]. We conclude that bariatric surgery might restore impaired irisin production but the effect depends on glucose and lipid metabolism. In some patients, irisin levels decreased despite successful body mass reduction.

Baseline serum adropin concentrations in the bariatric surgery patients and control participants were similar, but the concentration increased had significantly increased at 6 months in patients compared with controls. The changes of adropin serum levels after surgery were bidirectional, but unlike irisin and preptin, an increase was observed in $87 \%$ of the patients. The results suggest that obesity may not impair adropin production and that body mass reduction may increase production, enhancing the effect of surgery. The results is in line with a previous report of increased serum adropin after body mass reduction with 8 weeks of exercise training in obese elderly people [22] and 3 months after Roux-en-Y gastric bypass in overweight and obese adults [19]. Patients with an increase in serum adropin after surgery were younger than those with a decrease, but serum adropin was not significantly correlated with age as reported by Butler et al. who reported a negative correlation of adropin serum levels and age [19]. The relationship of age and serum adropin requires additional study.

The changes in serum preptin were similar to those of adropin. Although comparable to controls before surgery, preptin increased in the 6 months after surgery, but with borderline significance. Like irisin and adropin, change in preptin level after surgery was bidirectional. Multiple regression analysis found that EBW and BMI at 6 months were independently associated with serum preptin level, but the association as not significant when all patient characteristics were evaluated together. To the best of our knowledge, there are no other studies on the changes in preptin production after body mass reduction. Postoperative changes in preptin need further investigation in a larger sample size over a longer period of observation.

The changes of serum irisin, preptin, and adropin were not related to the type of bariatric surgery; the baseline and follow- 
up results in patients with LABG and LSG before and after surgery were similar. Irisin, adropin, and preptin levels were higher 6 months after bariatric surgery than at baseline. The changes in each of the three peptides were bidirectional. We conclude that in some patients, body mass reduction may restore impaired production of energy regulating peptides that may add to the benefits of the bariatric procedure. Studies are ongoing to assess the changes at 12 and 24 months after surgery to determine whether the changes associated with the reduction of body mass are transient or permanent.

Acknowledgments The authors gratefully acknowledge technical assistance by Mirosława Kasprzak.

Funding Information The study was supported by grant KNW-1-195/K/ 6/K from the Medical University of Silesia.

\section{Compliance with Ethical Standards}

Conflict of Interest The authors declare that they have no conflict of interest.

Informed Consent Informed consent was obtained from all individual participants included in the study.

Statement of Human and Animal Rights All procedures performed in studies involving human participants were conducted in accordance with the ethical standards of the institutional and/or national research committee and with the 1964 Helsinki declaration and its later amendments or comparable ethical standards.

Open Access This article is distributed under the terms of the Creative Commons Attribution 4.0 International License (http:// creativecommons.org/licenses/by/4.0/), which permits unrestricted use, distribution, and reproduction in any medium, provided you give appropriate credit to the original author(s) and the source, provide a link to the Creative Commons license, and indicate if changes were made.

\section{References}

1. So B, Kum HJ, Kim J, et al. Exercise-induced myokines in health and metabolic diseases. Integr Med Res. 2014;3:172-9.

2. Aydin S. Three new players in energy regulation: preptin, adropin and irisin. Peptides. 2014;56:94-110.

3. Barbatelli G, Murano I, Madsen L, et al. The emergence of coldinduced brown adipocytes in mouse white fat depots is determined predominantly by white to brown adipocyte transdifferentiation. Am J Physiol Endocrinol Metab. 2010;298:E1244-53. (14)

4. Boström P, Wu J, Jedrychowski MP, et al. A PGC1- $\alpha$-dependent myokine that drives brown-fat-like development of white fat and thermogenesis. Nature. 2012;481(7382):463-8. (15)

5. Xin C, Liu J, Zhang J, et al. Irisin improves fatty acid oxidation and glucose utilization in type 2 diabetes by regulating the AMPK signaling pathway. Int J Obes. 2016;40:443-51. (17)

6. Timmons JA, Baar K, Davidsen PK, et al. Is irisin a human exercise gene? Nature. 2012;488:E9-E10. (16) 
7. Buscemi S, Corleo D, Vasto S, et al. Factors associated with circulating concentrations of irisin in the general population cohort of the ABCD study. Int J Obes. 2018;42(3):398-404.

8. Oelmann S, Nauck M, Völzke H, et al. Circulating irisin concentrations are associated with a favourable lipid profile in the general population. PLoS One. 2016;11(4):e0154319.

9. Gouda W, Mageed L, Shaker Y, et al. Assessment of serum vitamin D and irisin levels in obese patients. Clin Lab. 2018;64(11)

10. Sahin-Efe A, Upadhyay J, Ko BJ, et al. Irisin and leptin concentrations in relation to obesity, and developing type 2 diabetes: a cross sectional and a prospective case-control study nested in the Normative Aging Study. Metabolism. 2018;79:24-32.

11. Demirpence M, Yilmaz H, Colak A, et al. The effect of sleeve gastrectomy on serum irisin levels in patients with morbid obesity. Endokrynol Pol. 2016;67:481-6.

12. Shoukry A, Shalaby SM, El-Arabi Bdeer S, et al. Circulating serum irisin levels in obesity and type 2 diabetes mellitus. IUBMB Life. 2016;68(7):544-56.

13. Fukushima Y, Kurose S, Shinno H, et al. Effects of body weight reduction on serum irisin and metabolic parameters in obese subjects. Diabetes Metab J. 2016;40(5):386-95.

14. Majorczyk M, Staszkiewicz M, Szklarczyk J, et al. The influence of bariatric surgery on serum levels of irisin and nesfatin-1. Acta Chir Belg. 2018:1-7.

15. Lee YJ, Heo Y, Choi JH, et al. Association of Circulating Irisin Concentrations with weight loss after Roux-en-Y gastric bypass surgery. Int J Environ Res Public Health. 2019;16(4):E660. https://doi.org/10.3390/ijerph16040660.

16. Liu YS, Lu Y, Liu W, et al. Connective tissue growth factor is a downstream mediator for preptin-induced proliferation and differentiation in human osteoblasts. Amino Acids. 2010;38(3):763-9.
17. El-Eshmawy M, Abdel Aal I. Relationships between preptin and osteocalcin in obese, overweight, and normal weight adults. Appl Physiol Nutr Metab. 2015;40(3):218-22.

18. Mierzwicka A, Bolanowski M. New peptides players in metabolic disorders. Postępy Hig Med Dosw (Online). 2016;70:881-6.

19. Butler AA, Tam CS, Stanhope KL, et al. Low circulating adropin concentrations with obesity and aging correlate with risk factors for metabolic disease and increase after gastric bypass surgery in humans. J Clin Endocrinol Metab. 2012;97(10):3783-91.

20. Huh JY, Panagiotou G, Mougios V, et al. FNDC5 and irisin in humans: I. predictors of circulating concentrations in serum and plasma and II. mRNA expression and circulating concentrations in response to weight loss and exercise. Metabolism. 2012;61(12): $1725-38$.

21. Du XL, Jiang WX, Lv ZT. Lower circulating irisin level in patients with diabetes mellitus: a systematic review and meta-analysis. Horm Metab Res. 2016;48(10):644-52.

22. Fujie S, Hasegawa N, Kurihara T, et al. Association between aerobic exercise training effects of serum adropin level, arterial stiffness, and adiposity in obese elderly adults. Appl Physiol Nutr Metab. 2017;42(1):8-14.

Publisher's Note Springer Nature remains neutral with regard to jurisdictional claims in published maps and institutional affiliations. 